Cahiers de recherches médiévales

\title{
Vivre ensemble selon des règles communes
}

Difficultés, discussions et révoltes en Berry médiéval (XIII ${ }^{\mathrm{e}}-\mathrm{XV}^{\mathrm{e}}$ siècles)

Françoise Michaud-Fréjaville

\section{CpenEdition}

Journals

Édition électronique

URL : https://journals.openedition.org/crm/1552

DOI : $10.4000 / \mathrm{crm} .1552$

ISSN : 1955-2424

Éditeur

Honoré Champion

Édition imprimée

Date de publication : 15 mars 2003

Pagination : 29-52

ISSN : $1272-9752$

Référence électronique

Françoise Michaud-Fréjaville, "Vivre ensemble selon des règles communes », Cahiers de recherches médiévales [En ligne], 10 | 2003, mis en ligne le 04 octobre 2007, consulté le 15 décembre 2022. URL http://journals.openedition.org/crm/1552 ; DOl : https://doi.org/10.4000/crm.1552

Ce document a été généré automatiquement le 15 décembre 2022.

Tous droits réservés 


\title{
Vivre ensemble selon des règles communes
}

Difficultés, discussions et révoltes en Berry médiéval (XIII ${ }^{\mathrm{e}}-\mathrm{XV}^{\mathrm{e}}$ siècles)

\author{
Françoise Michaud-Fréjaville
}

1 On ne saurait concevoir le proche voisinage de groupes humains sans un minimum de règles et d'usages connus et acceptés de chacun. Mais aux observateurs lointains que sont les historiens il échappe l'immense majorité des accords, non seulement tacites mais parfois aussi dûment rédigés, dont la mémoire plus que les preuves faisait tenir ensemble à peu près en paix hameaux et villages médiévaux. Souvent c'est la rupture du consensus entre rustiques et maîtres, doublée de conflits entre les paysans euxmêmes, qui révèle les forces et les faiblesses des groupes humains au sein des terroirs. Les tensions aboutissent trop souvent, on le sait, à des excès de gestes et de paroles, c'est l'aubaine du chercheur qui, après d'ennuyeux dépouillements d'actes et de listes terre après terre, cens, tailles, achats et ventes divers, se réjouit de trouver le copieux procès et parfois la belle lettre de rémission. Enfin nos villages s'animent, les bouches s'ouvrent, les témoins parlent, les clans apparaissent au grand jour, tout le monde ment plus ou moins, des pressions s'exercent, parfois les coups s'échangent et le sang coule, et pour rétablir l'ordre public, la paix sociale et la sécurité de tous, il faut en passer par des concessions réciproques. Alors l'usage ancien est modifié, les nouveautés insupportables sont corrigées. Les caractères de la communauté villageoise changent en partie par l'introduction ou l'abandon par voie de justice d'habitudes, de prestations, de facilités ou d'entraves qui obligent les individus et les groupes à s'organiser autrement, à rétablir un nouvel équilibre. On ne répétera jamais assez que le monde rural n'est en rien statique, incapable d'adaptation, ni routinier.

2 Dans le Berry qui a occupé une bonne partie de mes recherches fort peu de villages étaient régis par des textes originaux : le quart nord-est suivait largement la coutume de Lorris sans que les adaptations proprement locales nous soient toujours perceptibles, les autres suivaient des coutumes particulières que seule la volonté opiniâtre des juristes a réussi à dire « de Berry » entre XIV et XVI ${ }^{\mathrm{e}}$ siècles ${ }^{1}$. Le caractère remarquable de celle Lorris était la liberté de l'ensemble des communautés au sein de 
laquelle elle s'exerçait, à l'inverse, celle de Berry admettait les diversités des conditions personnelles comme possibles en un même lieu, maintenait la coexistence du servage et de la liberté pour des populations amenées à vivre porte à porte sous des règles seulement partiellement communes. Cet aspect sera abordé en premier ${ }^{2}$. Les nouvelles conditions démographiques, la remise en valeur de terroirs et la nécessaire réévaluation des relations entre les possesseurs du sol et les exploitants à la fin du $\mathrm{XV}^{\mathrm{e}}$ siècle ont amené de violentes tensions qui ne sont qu'un des aspects d'une adaptation des communautés et des individus à des conditions sociales et politiques inédites ${ }^{3}$. Ce que ces troubles, d'importance inégale, révèlent des raideurs défensives et de l'intelligence du moment fait l'objet de l'essentiel de mon propos.

coutumes telles qu'elles avaient été concédées en Berry étaient dans l'ensemble vieillies, voire obsolètes au XIV ${ }^{\mathrm{e}}$ siècle, et cependant on se contenta largement par la suite de les renouveler avec quelques aménagements mais sans en accroître réellement le nombre. Il conviendrait d'abord - ce qui nous éloignerait trop de notre propos - de regarder d'assez près si les rédactions primitives étaient véritablement des manumissions complètes et si les modifications successives ont entraîné des conséquences sur les conditions des personnes et sur les rapports entre les paysans. Beaucoup des concessions des $\mathrm{XII}^{\mathrm{e}}$ et XIII ${ }^{\mathrm{e}}$ siècles furent faites, en vérité, sans que la servitude en général, dont le terme même est fort rarement présent, soit remise en cause. Guillaume de Lignières a bien reconnu que les gens du castrum de Lignières et du bourg Saint-Hilaire qui le flanquait étaient in plena libertate, Pierre de Graçay a certes affirmé qu'il délivrait ses dépendants de la ville de Graçay ab omni jugo servitutis ${ }^{5}$, et dans sa confirmation le roi Louis IX rappela fermement qu'il s'agissait d'une manumission complète ${ }^{6}$. Ces formules sont très exceptionnelles. Bien des "libertés » accordées, par ailleurs, touchaient à des contraintes qui n'étaient pas recognitives d'une sujétion des personnes mais concernaient des droits de justice (pas de procès hors du lieu, prises de bêtes, amendes forfaitaires), de ban pesant sur tous (guet, service militaire ${ }^{7}$, four et moulin seigneuriaux), ou de propriété éminente du sol du seigneur, tels des droits d'usage liés le plus souvent à la pure localisation géographique de l'habitat et non à la condition des tenanciers, comme nous le verrons plus loin. S'il subsistait presque toujours quelques corvées de foins ou de transport du vin, rares étaient expressément citées des tailles abonnées ${ }^{8}$ ou aménagées ${ }^{9}$, recognitives de servitude, pas plus que les «tailles aux quatre cas» qui ne l'étaient pas ${ }^{10}$. Cela n'éclaire pas la situation de la majorité des villageois du Berry. Tout bien pesé, je ne peux assurer de véritables affranchissements avant 1300 qu'à Châteauneuf-sur-Cher (1265), Lignières (1268), Graçay (1278), Saint-Palais (1279). Il faut y joindre les deux Septaines de Bourges et Dun-le-Roi, avec la réserve de toutes les enclaves des seigneuries et les droits de suite qui s'y exerçaient. Il me semble qu'en Berry se pose fortement pour l'historien, mais dans une problématique largement inverse, le problème déjà évoqué en Vermandois Champagne et Flandres par R. Fossier : « là où le servage subsiste, les serfs sont proches des libres " ${ }^{11}$ : ici, là où la liberté existe avant le XIV ${ }^{\mathrm{e}}$ siècle, les libres restent proches des serfs.

La délimitation de l'espace de la franchise par la désignation du castrum ${ }^{12}$ ou du bourg ${ }^{13}$, par des croix ${ }^{14}$, des fossés, une limite ${ }^{15}$, un circuit précis ${ }^{16}$ donnait à ceux qui avaient une résidence permanente dans l'enceinte virtuelle ainsi dessinée - et pour eux seuls ${ }^{17}-$ le plein exercice des libertés octroyées, tout comme, peut-être, l'obligation des devoirs 
généralement réaffirmés. Mais je ne vais pas aussi loin que G. Devailly qui voit dans les chartes du XIII e siècle "l'assurance de la liberté personnelle " ${ }^{18}$. Si le texte qui nous a été transmis ne spécifie pas la suppression des limites au formariage et au remariage des veuves, l'aménagement, la diminution ou la disparition de la mortaille (ou de l'échoîte), l'élimination des tailles serviles, à volonté ou abonnées, au profit de droits de bourgeoisie, la possibilité du déguerpissement sans droit de suite du maître ni récupération des biens, l'accès à toutes les fonctions, alors nous sommes encore en pays de servitude. Or les droits de suite, en particulier, furent très fortement réaffirmés aux $\mathrm{XIV}^{\mathrm{e}}$ et $\mathrm{XV}^{\mathrm{e}}$ siècles dans les dénombrements seigneuriaux comme dans les faits. Les partages d'enfants en sont la preuve et on retrouve ceux-ci dans tous les fonds d'archives de lieux relevant de la coutume de Berry ${ }^{19}$. Le passage à la véritable liberté, quand il s'est fait au Moyen Âge, fut tardif et limité dans l'espace.

Des représentants permanents des communautés sont assez rarement présents dans les chartes berrichonnes. Sous des noms divers (boni viri, probi homines, electi, « cossorts ") on ne les rencontre que dans une vingtaine de localités, sans aucune concentration géographique significative. Leurs attributions sont très inégales et en général limitées à la confection de l'assiette des versements annuels ou exceptionnels. Quelquefois ils sont commis à la garde des biens des gens momentanément partis ou tombés en déshérence en attentant les délais coutumiers (Châteauneuf ${ }^{20}$ ), et parfois réduits à la seule tenue des listes du guet (Charost $\left.{ }^{21}\right)$. Désignés par les chanoines de la Cathédrale à Beaulieu-surLoire ${ }^{22}$, ils sont élus par la communitas dicte libertate de Châteauneuf-sur-Cher, par la communitas villae des Aix-d'Angillon ou le consilium villae de Mennetou-sur-Cher, savoir si le consilium est tout ou partie de la communitas reste en question. Le mode précis selon lequel ils sont choisis n'est la plupart du temps pas défini. Le terme electus sans mention d'électeurs ne préjuge bien sûr d'aucun scrutin et peut être une désignation sur liste par le seigneur. Le cas de Bengy-sur-Craon, avant de voir plus loin celui de la seigneurie de Graçay, mérite pourtant d'être relevé, car un examen même minutieux ne permet que de faire une lumière bien faible.

Bengy-sur-Craon : paysans, communauté et répartition des charges.

Le texte que La Thaumassière inséré dans Les coutumes locales ${ }^{23}$ n'est pas une charte des libertés en forme mais la copie d'un compromis, ou d'une transaction comme le dit le compilateur, entre le chapitre cathédral de Bourges et les habitants de la paroisse de Bengy. Sise en champagne de Bourges, la " prévôté » de Bengy était un des joyaux de la fortune canoniale ${ }^{24}$, et la discussion survenue en 1257 n'est pas un signe avant-coureur des difficultés économiques à venir mais d'une certaine prise de conscience des dépendants de la seigneurie de la pression qu'ils pouvaient exercer sur leurs seigneurs. Jusqu'alors les devoirs s'exerçaient essentiellement sous la forme des charrois ${ }^{25}$. En 1228 , premier compte canonial conservé, on dénombrait 38 à 40 feux qui labouraient à la houe ou à la charrue et devaient trois charrois annuels et des avenages, mais à côté s'alignaient 29 nouvelles exploitations qui relevaient d'une autre forme de perception, les novalia en fait des terrages, et étaient dispensés des charrois. Cet aménagement de date inconnue, destiné à attirer des exploitants et à remplir les greniers canoniaux dut amener des tensions entre les Bengiacois. En 1257, on était encore en pleine expansion démographique, on misait sur plus de trois cents feux ${ }^{26}$ et il semblait aux habitants qu'une taxe unique par répartition remplaçant les deniers et avenages de coutume leur serait plus favorable. 
7 Les habitants, humbles et tenaces quémandeurs (cum humiliter et magna instancia rappelle le texte sous-entendant évidemment une tension certaine entre les parties), se firent représenter auprès de l'archevêque, garant de la transaction, par sept procureurs, dûment mandatés. Nous ne savons pas selon quelles modalités sinon que le choix se fit selon la « volonté et l'accord » à l'unanimité des hommes de la paroisse ${ }^{27}$. On ne peut affirmer que la sélection se fit lors d'une convocation dans l'église ou à l'issue d'une messe dominicale, ni que tous les hommes étaient présents. On doit tout au moins noter qu'au nom du procureur Drouin Bochard (Droynus Bochardi de Charpaignes) est accolé le lieu de Charpeigne, un gros hameau sur la route de Bengy à Dun, ce qui incite à penser que ce premier choix se voulait aussi représentatif des écarts de la paroisse. Ces hommes discutèrent avec l'archiprêtre de Germigny commis à l'arbitrage par l'archevêque Philippe Berruyer ${ }^{28}$. Le règlement qui s'en suivit utilisa une répartition par hameaux pour les sept hommes de confiance élus chaque 24 juin et qui devaient établir l'assiette des soixante-quinze livres parisis destinées à remplacer de manière forfaitaire la taille, les charrois, le fouage, les avenages, la corvée, la mortaille ${ }^{29}$. Au-delà de 300 feux, chaque feu supplémentaire serait taxé cinq sous, mais on n'irait pas plus loin que 100 sous de plus; en deçà le forfait devait être réparti sur tous selon les moyens de chacun. Deux boni viri venaient du chef-lieu et les cinq autres de quatre hameaux. Les chanoines se réservaient le droit, en cas de choix douteux ou discordant ce qui supposait un véritable débat avec vote puisqu'on envisageait une élection à la majorité ${ }^{30}$, ou si les élus ne faisaient pas leur travail dans les délais ${ }^{31}$, de choisir euxmêmes les répartiteurs. La méfiance entre les parties était suffisante pour que l'on ait prévu un rôle annuel en trois exemplaires dont l'un détenu par ces prud'hommes, l'autre par les chanoines et le troisième conservé par le prévôt du moment des chanoines, afin d'éviter une fraude préjudiciable, ne pateat locus fraudi! La somme réunie devait être versée le 22 juillet, à la Sainte-Madeleine, on le voit un court délai d'un mois, en pleine saison des moissons, les répartiteurs devaient donc appartenir au niveau supérieur des paysans, être des personnages susceptibles de distraire quelques jours pour se consacrer à leur tâche administrative. Il y avait dans les deux termes calendaires de la mission des procureurs, fixés avec subtilité, une sorte d'entente tacite : les chanoines préféraient discuter avec les familles les plus puissantes comme mieux aptes à saisir les intérêts des deux parties, les dépendants envoyaient ceux qui pouvaient avoir un certain poids auprès des maîtres et les plus riches familles acquéraient officiellement un rôle local dirigeant. Enfin l'apparition du prévôt seigneurial dont c'est la seule mention dans la « paix et convention » de 1257, introduit le rappel d'un autre intermédiaire local entre paysans et chanoines.

8 Au-delà des apparences il faut se demander si cet accord avait véritablement libéré les hommes. De toute façon, selon les clauses de la transaction, les gens de Bengy en raison de leurs biens restaient liés aux chanoines. Comme dans d'autres seigneuries la faculté de tester, de léguer, de donner en douaire ou en dot les biens immobiliers situés dans la seigneurie demeurait conditionnée à la légitimité (les bâtards sont exclus, sinon de quelques meubles), à la résidence dans la paroisse et à l'appartenance du nouveau détenteur à la condition du seigneur, en cas contraire après un an et un jour il fallait revendre le bien à un ou une paroissienne effectivement sous la loi des chanoines. Le formariage, totalement licite, ne pouvait en aucun cas justifier de la non-résidence des tenanciers, on était libre d'épouser hors de la seigneurie mais pas d'exploiter de l'extérieur. Tous ceux qui partaient en quelque façon sous le dominium d'un seigneur 
extérieur ne pouvaient se placer sous un pouvoir inférieur, égal ou supérieur sans l'accord formel des chanoines, sous peine de la saisie de leurs biens à Bengy si aucun héritier ne pouvait en recevoir la dévolution. Cela supposait, par exemple, ne pouvoir devenir bourgeois du roi sans permission.

C'était une liberté effectivement fort limitée ${ }^{32}$. Mais elle aurait pu être compensée, au temps du «monde plein » par la légèreté relative de la charge. Or dans la comptabilité des chanoines de Saint-Étienne de 1301-1302, on trouve mention pour Bengy de la "grande taille» (pro magna tallia) de 70 livres parisis et de la «taille des francs" (pro tallia francorum) comptée pour 50 sous parisis ${ }^{33}$. Le détail des noms n'est pas donné, nous n'avons pas les moyens de savoir quels sont les individus et les lieux concernés par l'une et l'autre imposition. La base de 75 livres pour 300 feux estimés revenait à taxer à 5 sous en moyenne, ce que rappelaient les 5 sous par feu supplémentaire. On voit qu'en 1302 les chiffres ne correspondent même pas aux 75 livres de l'accord et on ne trouve nulle trace des 7 sous de l'amende prévue pour les mauvais payeurs. Visiblement quelque chose n'a pas fonctionné. En dépit des espérances, le nombre des habitants fut insuffisant pour que l'abonnement soit favorable aux chanoines qui ne touchaient même pas la totalité espérée. La charge pesait trop lourdement sur des paysans désormais de plus en plus réticents.

En 1334, un procès opposa les habitants et leurs seigneurs, il semble que ces derniers aient voulu faire participer leurs dépendants à une aide royale. Les gens de Bengy se défendirent, ils donnèrent à leurs sept élus une procuration pour présenter leurs doléances, mais ne purent se dégager de cette affaire qu'en ajoutant vingt livres annuelles au forfait et en obtenant l'assurance de ne pouvoir être taxés que pour le bien exprès du chapitre: pro augmentatione, vel defensione ecclesie, vel libertate, vel terre melioratione, vel casu enorma dampna ${ }^{34}$. Cela ne leur épargna pas le subside royal de 1338 ainsi que nous l'apprend une correspondance entre les bureaux royaux et ceux du receveur royal de Bourges. On mandait en effet depuis Paris de s'enquérir si les « habitans de Bangy en la prevosté de Bourges laquelle ville est au chapistre de Bourges sont taillaibles a volente dudit chapistre ou abonnes ", le receveur répondit qu'ils étaient effectivement abonnés depuis trois ans à vingt livres parisis supplémentaires et qu'au surplus on avait déjà levé sur eux vingt livres tournois "pour la présente guerre ", somme qu'ils pouvaient d'ailleurs bien supporter, ajoutait-il crûment. La franchise de Bengy était-elle la liberté ou une liberté ? Le receveur royal semblait ne rien savoir d'un véritable affranchissement.

11 En tous cas l'arrangement ne pouvait tenir que si la somme était répartie sur un assez grand nombre de paroissiens. Au premier tiers du $\mathrm{XV}^{\mathrm{e}}$ siècle, le poids était devenu proprement intolérable en raison de la baisse de la population. Il fallut reconsidérer le paiement de ce qui était entre-temps bel et bien devenu un droit de bourgeoisie et en 1439 un nouvel accord " pour plus grande commodité ", abandonna le forfait pour fixer la redevance à 10 sous tournois de monnaie courante et 4 boisseaux de froment pour les plus riches, 6 sous 8 deniers tournois et 2 boisseaux de froment pour la catégorie intermédiaire et 3 sous tournois et un boisseau pour les plus pauvres, l'acte modificateur cite 110 personnes ayant participé à la procuration donnée à deux d'entre eux, soit donc trois fois moins que les feux espérés en $1257^{35}$. On a également la preuve d'une société villageoise où les différences de richesse sont bien nettement accusées et en quelque sorte entérinées. Nous avons la recette de cette taille pour 1444-1445 et quelques autres années très espacées, elle était incluse pour les boisseaux de froment 
dans les comptes de la graineterie de Bengy qui ne sont pas conservés en parallèle avec les comptes en deniers. Le rapport en 1445 est de moins de douze livres et il n'y a que trente et un taillables, un peu plus du tiers seulement des gens qui furent cités en 1439 pour donner procuration, les trois quarts se situaient dans la tranche inférieure ${ }^{36}$. Même en admettant une sous-évaluation habituelle en matière d'impôt, on mesure la charge insupportable que devait représenter l'abonnement de quatre-vingt livres parisis maintenu de 1338 à 1439. En tout état de cause il aurait fallu plus de cinq cents des plus pauvres pour honorer le forfait promis au début du XIVe siècle. Cette «taille " continua à être levée régulièrement dans les quelques registres conservés et si l'on voit les assujettis remonter jusqu'à une centaine de noms en 1481-1482, il ne se retrouve pas suffisamment de personne à Bengy à la fin du $\mathrm{XV}^{\mathrm{e}}$ siècle pour revenir à une somme forfaitaire qui eût paru avantageuse à la communauté des habitants ${ }^{37}$. Surtout, il me semble que la totale liberté des Bengiacois, en dépit de la présence des procureurs, n'est jamais clairement rappelée : taille abonnée, et transport des redevances à Bourges entretenaient encore à l'extrême fin du Moyen Âge une ambiguïté certaine dans les relations de la communauté avec ses seigneurs berruyers en cette frontière des deux coutumes serviles, celles de Berry et de Bourbonnais.

Paysans, communautés, libertés et seigneurs en pays de GraçayL'extinction des communautés serviles

La chronologie de la quasi-extinction du servage peut, au contraire, se préciser avec une certaine finesse en terre de $\mathrm{Graçay}^{38}$ : les receveurs des chanoines de la SainteChapelle de Bourges en ont tenu une comptabilité régulière, ce n'est pas de leur faute si le temps a conservé inégalement leurs registres. L'examen par décennies du nombre des tailles et des bourgeoisies du $\mathrm{XV}^{\mathrm{e}}$ siècle permet de voir qu'au sein d'une population qui s'est multipliée par trois et demi en trois quarts de siècle les évolutions sont divergentes. Le nombre de taxables est certes passé de 233 à 848 chefs de familles, mais si l'on constate une remarquable hausse du nombre des hommes libres après le milieu du XV $\mathrm{X}^{\mathrm{e}}$ siècle (de 144 à 781 individus) on mesure simultanément, après l'embellie liée à un croît interne de reconstruction, une tout aussi rapide diminution de moitié des taillables entre 1446 et 1493 à la fois par rachats individuels et pas le jeu des mariages mixtes $^{39}$. Au cours de la première partie du $\mathrm{XV}^{\mathrm{e}}$ siècle, la proportion numérique des bourgeoisies et des tailles a tourné autour de deux tiers-un tiers, puis en vingt ans, entre 1460 et 1480, le nombre de taillables a diminué avec rapidité pour tomber à moins de $10 \%$ en 1481 (cf. tableau en annexe).

13 Le détail paroisse par paroisse non repris minutieusement ici montre cependant des disparités locales remarquables : aux portes de Graçay, chef-lieu de la seigneurie dont le dernier taillable disparaît après 1479 , la paroisse de Coulon conserve entre $40 \%$ et $45 \%$ de serfs parmi les 70 feux dépendants de la Sainte-Chapelle, Nohant-en-Graçay et Meunet-sur-Vatan ne descendent pas au-dessous de $20 \%$ de non-libres. À Parpeçay, zone pourtant assez marginale et qui pourrait échapper à la surveillance canoniale, des taillables font une nouvelle apparition en 1475 et 1492, peut-être justement en raison de la nécessité d'affirmer une dépendance paysanne envers les Messieurs de la SainteChapelle face aux hobereaux locaux. Trois paroisses limitrophes les unes des autres, aujourd'hui dans trois départements distincts, ont évolué à des rythmes différents : Genouilly $y^{40}$ est entièrement passée à la "bourgeoisie » entre 1466 et 1471, ses ultimes onze taillables devenant bourgeois en échange, apparemment, d'un abandon des usages des bois sans que, malheureusement, soit conservé l'acte libérateur ${ }^{41}$, alors que 
La Chapelle-Montmartin ${ }^{42}$ conserva un noyau « dur » de quatre familles serves jusqu'en 1485, pourtant un certain Vachier avait déjà acheté sa manumission en $1466^{43}$, les autres négocièrent comme à Genouilly et jugèrent vite avoir fait un marché de dupes ${ }^{44}$, dans les conditions qui seront rappelées plus loin. À Anjoin", les bourgeois dits " de Graçay " dont la condition personnelle nous paraît plus satisfaisante que celle des simples bourgeois ne s'accrurent que faiblement alors que les semi-serfs de la «franchise » de Vonnes résistèrent, en grande partie à cause d'une reconstitution du vignoble qui les avait favorisés ${ }^{46}$ et des usages des "Tailles » dites de Ruines. Ces derniers gens de Vonnes aussi protestèrent quand une "liberté » devenue apparemment la norme signifia l'expulsion des lieux d'usages et ils refusèrent comme les autres d'acquitter tailles et bourgeoisies ${ }^{47}$. C'est maintenant l'épisode agité qu'il nous faut examiner.

Entre Cher et Fouzon à la fin du $\mathrm{XV}^{\mathrm{e}}$ siècle, affirmation des communautés et individualisme paysan

14 En terre de Graçay, en 1372 au moment où le duc de Berry prit possession de la seigneurie, la liste des hommes payant les cens portant lods et ventes dans la paroisse de Genouilly, pour treize chesaux et cinq maisons villageoises, comprenait deux taillables serviles et sept bourgeois ${ }^{48}$; l'énumération des gens de La ChapelleMontmartin, pour dix-huit chesaux également aliénables, offrait onze taillables, plus de la moitié des détenteurs, mais aucun «bourgeois de Graçay », cela peut-être en raison d'un plus grand éloignement du chef-lieu et d'une politique d'accueil des officiers du duc Jean qui n'avait pas eu encore le temps de porter ses fruits ${ }^{49}$.

En résumé, au début du dernier quart du XIV ${ }^{\mathrm{e}}$ siècle quand Jean de Berry s'installa à Graçay, gros bourg plutôt que petite ville, les chesaux et les terres des libres et des bourgeois du duc ne semblaient pas se distinguer les uns des autres, ni d'ailleurs de ceux des hommes relevant de la quinzaine de seigneurs vassaux. Or les livres de gestion des officiers ducaux, poursuivis par les receveurs des chanoines de la Sainte-Chapelle, montrent que le regard porté par les chanoines et leurs administrateurs sur les rapports entre les conditions des hommes, leur dépendance seigneuriale et l'appropriation des terres s'est modifié assez sensiblement au cours des temps.

On voit au cours du $\mathrm{XV}^{\mathrm{e}}$ siècle seigneurs et exploitants discuter et tâtonner puis à Graçay se figer dans un système, se couler dans ce que nous appellerions un modèle, pour tenter de tirer les uns et les autres le maximum d'avantages des situations locales et ponctuelles en jouant sur toutes les arguties des textes et des coutumes ${ }^{50}$.

La seigneurie a accueilli de façon officielle en enregistrant leurs déclarations 480 nouveaux arrivants entre 1420 et $1465^{51}$. Beaucoup de ces gens ont fait souche, certains se déclaraient d'ailleurs in extremis, si l'on peut dire, à l'occasion de leur mariage. Après avoir fait aveu de bourgeoisie ils - ou elles - n'auraient guère dû se distinguer du reste de la population et leur indépendance primitive devait se fondre rapidement dans les routines de leurs nouvelles familles, ainsi que le montrent les généalogies où anciens et nouveaux occupants entrecroisent les mariages très fréquemment. Les seigneurs berrichons, comme bien d'autres dans le royaume tout entier ainsi que tant d'études locales l'ont montré ${ }^{52}$, ont cherché de façon toute naturelle à accroître le nombre de leurs dépendants et à augmenter le produit des redevances. Ils l'ont fait cependant d'une façon sensiblement différente de celle de leurs prédécesseurs des XII et $\mathrm{XIII}^{\mathrm{e}}$ siècles en privilégiant ces individus ou leurs communautés familiales, au 
détriment des regroupements collectifs villageois ; l'ère des villes neuves est bel et bien close. Les massifs incultes ou retournés à la lande ou à la forêt dégradée en raison des reculs démographiques, se sont repeuplés par des lots individuels avec habitat, souvent de grande taille, ce que l'on appelait depuis longtemps généralement en Berry les métairies, et non par la création de villages. Tout ceci figure aujourd'hui dans les manuels, tout comme le rappel des entraves mises un peu partout aux privilèges traditionnels des communautés ${ }^{53}$. Les exemples que je vais évoquer entrent parfaitement dans les normes connues des comportements réciproques des seigneurs et de leurs dépendants quand les pacages et les droits au bois furent en jeu, mais ce sur quoi l'accent doit être mis ici est le rôle joué par l'individu dans sa communauté : la paroisse, le village.

Une des premières manifestations des tensions en seigneurie de Graçay m'apparaît en 1448, non pas à l'occasion d'un affrontement entre les dépendants et leurs maîtres, mais d'une bien ordinaire affaire de limites entre les chanoines de la Sainte-Chapelle et le seigneur de Sauveterre, fief de la paroisse de Saint-Loup-sur-Cher ${ }^{54}$, Antoine du Bois, époux de Pernelle de Graçay. Il s'agissait simplement, si l'on peut dire car l'opération est en général délicate, d'un bornage entre les bois des deux seigneurs, à la limite des paroisses de Saint-Julien-sur-Cher, Saint-Loup-sur-Cher, La Chapelle-Montmartin, Dunle-Poëlier ${ }^{55}$ et Anjouin à proximité de l'ermitage de Sainte-Catherine-du-Feust, le bien nommé. Les parties avaient demandé chacune à six paysans du lieu de suivre les bornages avec les hommes de loi, il en vint onze sur douze ce qui est un bon rendement: la question était fort sérieuse. Les sergents et les hommes des paroisses firent leur travail en marquant les arbres au long des limites. Puis, lors de la visite finale par les seigneurs, le procès-verbal rapporte les âpres discussions qui avaient opposé les délégués commis au bornage : du côté des bois des seigneurs de Sauveterre les marqueurs des chanoines auraient empiété d'au moins deux arpents. Autant de moins pour les usages. Pour ne pas refaire tout le circuit « fust dit que pour si pou de choses ne demourroist pas l'accordt à faire ", et on en resta là. Un vidimus du compromis intervenu à propos des limites entre les seigneurs précisa plus tard que le bois en question mesurait cent quatre-vingt arpents carrés, soit à peu près cent soixante hectares du côté des seigneurs de Sauveterre ${ }^{56}$ sans doute le double du côté des chanoines. Il s'agissait en fait des bois et des usages sur les «tailles de Ruynes»-le nom modernisé en Ruines subsiste dans l'actuelle toponymie - dont bénéficiaient les habitants des hameaux serviles des paroisses mitoyennes, voisins auxquels s'ajoutaient les hommes de la franchise de Vonnes et ceux du prieuré proche de Coulommiers. Les uns et les autres à titres divers versaient des redevances d'usages forestiers :

"Aurilhages qui sont de tele condicion que quiconques demore es metes ou fins commancenz au lonc du ruau qui vient de l'estang de Nohant et chet en Foron et vait jusques a la franchise de Vonnes et puis en Gastine, a la Chapelle Moynemartin et a Genoilly et il y tiennent chievres ou pors, ils doivent pour chascun chep .i. den. t., si non les bourgois qui en sont quictes, et se paient on moys d'avril [...]

Pasquiers qui se paient a la Saint-Michel et sont de tele condicion que quiconques tient ou nourist pourceaulz es contrees, metes et fins ou le dessusdit aurilhage es dehu, excepté Gastine et la Chapelle Moynemartin, ilz doivent pour chascun chep de pors audessus de .i. an .iii. d., et au dessoubz de .i. an .i. d. se il ne tete, et ceulz qui tettent en sont francz ${ }^{57}$ »

19 Les habitants d'alentour et particulièrement ceux de la franchise de Vonnes avaient parfaitement raison de surveiller l'arpentage et de redouter que ce dernier soit suivi de 
près ou de loin d'un dépeçage des bois dont la conséquence signerait leur exclusion des zones d'usages et de pâturage. Au cours de la seconde moitié du $\mathrm{XV}^{\mathrm{e}}$ siècle, les habitants de la Chapelle-Montmartin commencèrent à solliciter des chanoines de Graçay le droit d'étendre leur terroir vers le sud-est, du côté des "tranchées » des religieux, en direction de ces tailles de Ruines dont une partie était dans leur finage le reste à Anjouin et Dun-le-Poëlier. La forme particulière de cette paroisse, une longue et étroite lanière, justifiait ce besoin d'espace. Les baux qui s'échelonnent depuis 1456 jusqu'à la crise de 1492 appartiennent à une série qui limite les mutations aux seuls dépendants, barons ou bourgeois, du seigneur, sous peine de " parisis pour tournois " en cas d'infraction ${ }^{58}$, et concernent des terres en «bois, bruyères et buissons ». Ces termes sont extrêmement importants : ils signifient selon la coutume locale que les pièces sont taillées dans des parties auparavant libres au pâturage, dans lesquelles il n'y avait pas de prise de bêtes ${ }^{59}$. Elles devenaient en revanche, du fait de ces baux, désormais appropriées par un particulier dans son intérêt personnel et dans celui des chanoines, au détriment des communautés. La construction d'une ou plusieurs maisons faisait partie du contrat, comme dit le bail à rente du 15 mai 1468 à Macé Salvet, un membre d'une famille présente dans la seigneurie depuis des générations, à propos des dix boisselées prises sur la «tranchée des chanoines » où il devait bâtir «pour seureté de la dite rente». Les précisions sur les maisons, le nombre de leurs fermes de charpente, la présence ou non d'une croupe (qui rendait plus difficile l'allongement des bâtiments selon les besoin) et les délais de construction de deux ans, permettent une vérification dans les listes de nouveaux cens des registres de comptes. On y lit que la plupart des contrats ont bien été remplis et qu'en particulier le terroir de la Marchoire, actuellement les Huets, à La Chapelle-Montmartin, tout comme une partie de celui des Tailles des Ruines à Dun-le-Poëlier ont été effectivement assez largement offerts à la culture entre 1466 et 1490 . Ainsi furent passés les baux de Guillaume et Jean Pichon dit Lore (11 octobre 1472$)^{60}$.

Les esprits commencèrent à s'échauffer dès 1488 , les pacages en usages se raréfiaient. Une vingtaine de nouvelles exploitations entrecoupaient les landes et buissons demeurant ouverts aux serfs et aux hommes des franchises ${ }^{61}$. Ceux-ci perdaient ces parcours presque gratuits qui leur permettaient non seulement de nourrir leurs propres bêtes mais aussi très certainement et discrètement des vaches et chevaux pris à cheptel à l'unité par des ententes entre particuliers, ce qui était illégal sur les communaux théoriquement réservés aux seuls habitants ayant feu et lieu pour leur propres bête. Les nouveaux occupants fermaient désormais leurs propriétés et lopins par les haies et des clôtures, pour se prémunir contre des pâtures illicites et les bêtes sauvages ("serfs (sic) et biches»), comme pour garder plus commodément les brebis et vaches de leur cheptel. C'est, sur une durée d'un quart de siècle, la gestation et l'éclosion du paysage «bocager » en Boischaut nord. Les chanoines et les seigneurs se réservaient de plus des espaces et garennes totalement en défens : le nom des " Parcs » est encore celui d'une partie de la zone boisée méridionale de la Chapelle-Montmartin.

21 En 1488, les chanoines offrirent à ceux des habitants de Genouilly, d'Anjouin et de La Chapelle qui n'étaient pas encore bourgeois de Graçay de les affranchir des tailles serviles, à la suite d'une discussion dont les détails nous sont inconnus, mais comprenait très certainement une clause discrète, voire obscure, de mise en défens ou de limitation à la pâture des bois canoniaux de Ruines et de Genouilly en échange de la diminution du droit de rachat de servage. 

preneurs. En janvier 1490 (n. st.) Jean Mesny alias Courtin et sa femme prenaient à perpétuité, avec les clauses usuelles des "parisis pour tournois ", dix-huit sextérées (soit dix-sept hectares) de ces « terres en bois et buisson [...] dans laquelle les homes de la serve condition de messeigneurs avoient leurs usaiges, mais ilz ont este manumiz et affranchis, moyennant qu'ilz ont renonce auxdits usaiges " ${ }^{62}$. On ne saurait être plus clair ! Les bois et buissons étaient lotis, les bois de réserves étaient fermés et défrichés : la liberté nouvelle, si peu différente sur bien des points de la servitude locale, n'avait fait qu'aggraver la situation matérielle des habitants. Les anciens serfs, ayant perdu leurs bois particuliers, devaient se rabattre sur les quelques bois et sur les vaines pâtures véritablement communs à tous, eux aussi en diminution. Davantage de bêtes, plus de ramassages sur moins d'espace pour une population en croissance démographique, tel était le résultat d'une « liberté » payée bien cher.

La violence du point de départ est certaine : Pierre Chippault de Poulaine, ne sut garder son calme quand Vincent Beaujean et Pierre Pichart entreprirent la plantation d'une haie en bordure de ses terres. D'injures en coups, il y eut mort d'homme. Chippault, dont la famille se partageait entre les chanoines de la Sainte-Chapelle et la puissante abbaye de Notre-Dame de Déols ${ }^{63}$ obtint une rémission royale à la fin de $1489^{64}$. L'affaire fit boule de neige: les habitants de la châtellenie de Graçay, après avoir arrêté le sergent de leurs seigneurs et l'avoir "sorti de son cheval ", puis obligé le capitaine de Graçay, Jean de Menou, venu les calmer et les «desesmouvoir ", à battre prudemment en retraite ${ }^{65}$, s'en furent trouver les officiers du bailliage, le prévôt royal et jusqu'à l'archevêque, leur représentèrent que les chanoines avaient "vendu la moitié » de leur forêt, que tous et chacun étaient victimes de la mise en défens des anciennes Tailles de Ruines et des bois de Genouilly et déclarèrent que désormais ils ne paieraient pas les fouages, ni ne feraient le guet puisque ces devoirs n'étaient plus suffisamment contrebalancés par des droits d'usage ${ }^{66}$. Le 6 septembre 1491 le Parlement de Paris accordait pourtant aux chanoines la levée de tous leurs droits habituels, mais leur interdisait jusqu'à comparutions des parties « d'empescher iceulx habitans d'avoir et mettre bestial aux dits bois et fourests ainsy quil est accoustumé de tous temps et anciennete en paiant par lesdits habitans les droiz et devoirs pour ce dehuz ${ }^{67}$. On ne pouvait pas mieux reconnaître qu'une contrepartie des tailles et fouages étaient bel et bien les droits d'usage. On ajourna les parties, hommes dépendants serfs et libres d'un côté, chanoines de l'autre et il fallut donc désigner des procureurs pour novembre 1491.

De paroisse en paroisse, 743 habitants dont nous avons les noms se rassemblèrent de place en olace pour élire des procureurs destinés à paraitre devant les officiers royaux et à suivre le procès en Parlement ${ }^{68}$. Sans détailler de façon énumérative, il est cependant intéressant de noter les nuances différenciant les dix-huit communauté concernées. Nous savons que pas une ne disposait d'élus prévus par des chartes dites de franchises, aussi fallut-il des modalités diverses. Dix villages utilisèrent une procédure préexistante en déléguant des fabriciens, la communauté se confondait alors avec les paroissiens. Ces fabriciens ne furent pas tous désignés pour l'occasion, c'est le cas par exemple à Nohant-sous-Graçay où Jean Chapploteau et Jean Hervier étaient marguilliers depuis décembre 1480 et à Orville qui n'avait pas jugé bon de renouveler son choix de 1469 ! Les autres semblent avoir tenu une assemblée paroissiale au cours de l'année 1490 en raison du conflit latent, ainsi La Chapelle-Montmartin (14 février

Cahiers de recherches médiévales, 10 | 2003 
1490 n. st.) et Luçay-le-Mâle - alors dit le Chétif - le 10 juin 1490. Les prêtres desservants assistèrent aux réunions, sauf à la Chapelle, à Luçay, à Avexy (aujourd'hui inclus dans Graçay) et à Parpeçay. Certaines assemblées eurent un cadre plus « laïque » si l'on ose s'exprimer ainsi: Saint-Oustrille près Graçay avait déjà désigné des « procureurs » en janvier 1479 (n. st.), Genouilly, peut-être pour l'affaire des derniers serfs, avait tenu assemblée et choisi ses procureurs le 12 juillet 1489 , on ne jugea pas nécessaire d'en tenir une nouvelle, Poulaines réunit la sienne le jour même où l'arrêt du parlement fut scellé (un Guillaume Chippault y fut élu procureur...) et Sainte-Cécile la convoqua pour le surlendemain. Quelques communautés usèrent du terme de «messagers especiaulx" comme les gens des «franchises de Vonnes" qui regroupaient, il est vrai, des individus de paroisses différentes, mais aussi ceux de Sembleçay et de Graçay même, comme pour souligner l'exceptionnelle procédure à l'égard des chanoines et réserver peut-être un statut moins juridique à leurs envoyés.

Ce qui est troublant est que le résultat de ces élections, soigneusement consigné, nom par nom, dans un registre ne coïncide pas toujours avec ce que le même registre a noté pour le récolement et l'examen des procurations qui eurent lieu du 20 au 25 novembre. De plus, le 26 novembre apparaissent lors de la comparution effective des fabriciens et autres procureurs de nouveaux noms de gens qui ne figurent sous aucune forme dans les listes précédentes! Ainsi pour Genouilly, deux seulement des six procureurs notés comme élus par l'assemblée présentèrent leurs procurations et deux procureurs présents devant le tribunal du bailliage nous arrivent avec un mandat non prévu et sans même avoir été enregistrés parmi les hommes réunis en assemblée. Il est encore heureux qu'un des deux ait pu être relevé parmi les censitaires de la comptabilité de la Sainte-Chapelle, sinon nous pourrions croire à une intrusion totalement extérieure aux intérêts en cause. L'apparente rigueur des longues énumérations et des dates précises ne pouvait masquer en réalité les pressions qu'exercèrent les deux parties en présence. On peut citer l'exemple de Giroux ${ }^{69}$, où l'assemblée du 30 mai 1490 a réuni 20 électeurs qui ont élu quatre fabriciens (Martin Guérin, Jean Millet, Renault Sauget Jean Vinau) sous le regard de Jean Berloie, prêtre. Or quand, seul des quatre, J. Vinau présenta sa procuration, avec lui se trouvaient Pierre Sauget, paroissien électeur mais non élu, qui à son tour produisit un document considéré comme valable, et Claude Peton qui surgit avec les deux autres sans avoir été présent à l'assemblée et encore moins élu. Or ce personnage était le fermier de l'importante métairie canoniale de Bois-Giroux, près de 60 hectares entre terres céréalières et bois ${ }^{70}$, un des derniers « barons ", c'est-à-dire non libres de la seigneurie, une puissance dans sa paroisse car il employait nombre de paroissiens comme hommes de bras. Sa présence fut-elle suscitée par les maîtres? De façon moins éclatante et nécessitant plus d'explication, des manœuvres apparaissent également du côté des paroissiens, comme à La Chapelle-Montmartin où un des Salvet, occupant des terres pourtant prises sur les «Tranchées des religieux " sans élection comme fabricien, son parent dûment élu le 14 février 1490 (n. st.), or ces Salvet se révélèrent par la suite les meneurs de l'opposition aux religieux. Les représentants des paroisses étaient donc plus vigilants qu'on peut croire : certains avaient déjà protesté que dans certains cas on n'avait pas procédé à un ajournement par procurateurs en règle mais par «seulement troys ou quatre particulliers de chacune paroisse combien quilz ne fussent procureurs d'icelles ». En fait, les entorses provenaient des deux côtés. 
26 L'arrêt imposé par le Parlement et signifié aux procureurs ne donnait pas la solution pour éviter de nouveaux troubles, il appliquait simplement de façon conservatoire les modalités du siècle précédent, ce «temps du duc», dont les parties avaient fourni les registres. C'était un statu quo pour les personnes, devant lequel les habitants s'inclinèrent, mais les haies déjà plantées restaient en place, les surfaces laissées aux usages n'avaient pas augmenté d'un arpent, l'avenir n'était en rien garanti pour les paysans que des règles communes ne protégeaient plus.

L'affaire monta cependant au parlement de Paris. Les éléments des plaidoiries des deux parties que nous connaissons uniquement par la longue liste argumentaire des seuls chanoines reposaient largement non sur la mémoire des habitants et des seigneurs ou les coutumes générales de Berry mais sur les preuves fournies par les registres « du temps du duc ». Selon les dépendants, ces derniers démontraient clairement que les bourgeois et les barons avaient bien le droit au bois et au pacage des porcs. Les chanoines étaient d'un avis contraire: faute de gens les anciennes terres exploitées étaient devenues friches mais n'étaient pas des usages traditionnels, les listes de nihil en faisaient foi, et de plus, les habitants avaient racheté récemment leur condition au prix de l'abandon de droits dont des registres plus que trentenaires prouvaient en outre clairement la totale désuétude. L'argument de la violence fut une nouvelle fois tenté par les habitants. S'agissait-il de faire revenir les chanoines à l'état antérieur? Sans doute pas, mais plutôt de les obliger à prendre en compte un rapport de force nouveau: la masse numérique des exploitants libres désireux eux aussi de pénétrer dans les défens.

On trouvera en annexe le récit enregistré par le greffe du bailliage de Berry du soulèvement de janvier 1492 (n.st.) mené par quelques individus, les Salvet en particulier, qui pourtant avaient les premiers ou presque, on l'a vu, bénéficié des baux de bois et buissons, mais devaient se sentir menacés par la concurrence ${ }^{72}$. Parmi la vingtaine de conjurés on retrouve comme instigateur Jean Garnier le jeune qui fut un fabricien-procureur en novembre 1491 et comme acteurs les Beault, Besart, De Bourges, Thierry, anciens participants à l'assemblée électrice fort restreinte de la ChapelleMontmartin (25 hommes dont 22 relevaient de la seigneurie de Graçay) de novembre 1490. Groupe du cœur paroissial, certes, mais aussi rassemblements familiaux de frères et cousins. Les gens de la Chapelle et quelques-uns de Genouilly, armés dirent-ils de leurs seuls outils, fourches, épieux et cognées, allèrent défoncer les haies des nouvelles exploitations marginales. Les quatre que l'on arrêta et mena en deux étapes vers Bourges furent délivrés à Preuilly, la première nuit, par un coup de force complice des autres, avec voies de fait et mauvaises paroles envers les autorités et même lèsemajesté envers le roi et la reine, moqués dans leur devoir de sauvegarde des biens canoniaux, y compris à Preuilly terre relevant également de la Sainte-Chapelle.

Qui étaient vraiment les meneurs? Il faut lire de près tous les textes pour comprendre que les pauvres gens entre "Cher et Fouzon" avaient de leur côté des capacités d'action: Étienne Salvet s'était rendu en personne à Paris! L'hiver on dispose de davantage de temps sans doute, mais il y avait également l'oreille et la parole d'un conseil. Ce dernier avait anticipé pour les Berrichons l'annonce d'une décision en leur faveur du Parlement, rien de moins ! Dans leur argumentaire les seigneurs de la SainteChapelle ne se privent pas d'évoquer un de ces "conseils ", malfaisant et donc poussé au noir caricatural. Il s'agit de maître Jean Courault, le propre curé de la ChapelleMontmartin, qui est à leurs yeux l'origine des assemblées illicites, des audacieuses 
expéditions punitives contre les haies et les clôtures, les maisons neuves et leurs habitants. Faute de vouloir reprendre les arguments de ce clerc, lesquelles risqueraient peut-être de tourner en leur défaveur, les chanoines n'hésitent pas à forcer le trait ad hominem : «il est toute commune renommee et tout notoire qu'il estoit passé a cheval par la ville de Grassay et avoit derriere luy une paillarde [...] et a bien monstré a ses paroissiens qu'il est ung foul ». De ce personnage, le greffier de Bourges, dans son récit de l'émeute la Chapelle-Montmartin, ne dit mot mais il note que, lors de la tentative de récupération à Preuilly, des coupables de l'expédition contre les "rentiers" des chanoines, figure un certain maître Étienne Dupont, autre personnage auquel le nom de maitre accorde un statut nettement supérieur à celui des simples paysans des paroisses. Ce maître semble avoir négocié avec le sergent royal ou couvert la fuite de trois des quatre prisonniers. Et voici donc au moins deux « autorités » pour aiguiser et mettre en forme les justifications des dépendants. La cohésion des paysans a cependant des limites. L'interrogatoire de Pierre Garnier l'aîné, trop dur d'oreille, grâce à Dieu, pour avoir entendu ou vu quoi que ce soit de vraiment compromettant à Preuilly, lui permet d'éluder les "conseils», de revendiquer l'initiative propre de ses concitoyens, de refuser avec vertu l'insolence envers les chanoines et encore davantage la désobéissance envers le roi, de nier une précédente expédition contre des charbonniers acheteurs du bois accaparé par les chanoines. Les dénégations rendent encore plus vraisemblables l'échauffement des paroles et l'anticléricalisme verbal des paroissiens, furieux contre leurs seigneurs, sûrs de leurs droits. Les clivages entre les familles et les intérêts se révèlent dans les choix que fait Jean Garnier l'aîné du refus de la confrontation avec l'autre moitié de la paroisse, où curieusement apparaissent alors les femmes et veuves supposées détenir la mémoire locale : les Gallier, les Moireau et les Pichon dit Lore ne sont point de ses amis, et c'est un euphémisme. Les Gallier et Pichon, les Mesny sont des nouveaux venus, de la deuxième génération nous disent les généalogies pour le compte des chanoines, les Garnier sont des anciens du terroir, qui ont été les dépendants des Graçay, puis du duc Jean et enfin des "Vénérables". Devons-nous croire à des haines irrémédiables?

L'affaire de Preuilly ne semble pas avoir eu de suite grave, si l'on en croit leurs avocats ces messieurs de Bourges faisaient souvent preuve de la plus grande mansuétude y compris envers les voies de fait et les coupes frauduleuses ${ }^{73}$. De fait, les chanoines avaient gagné : la comptabilité, faute de la sentence définitive du Parlement, montre que les aurilhages et pasquiers avaient bel et bien disparu dès la seconde moitié du $\mathrm{XV}^{\mathrm{e}}$ siècle. Le 28 juin 1500, Jean et Thomas Pichon dit Lore, Jean Garnier, Étienne Gallier, quatuor naguère si dissonant et désormais bien accordé, en compagnie de cinq autres hommes de La Chapelle-Montmartin et de Chabris prenaient à bail 33 ha de la "Tranchée de messeigneurs», jouxe les usages de Chabris ${ }^{74}$. À cheval sur deux paroisses, dans l'éloignement économique vis-à-vis des deux communautés, les fauteurs de trouble assagis occupent désormais des espaces qu'ils se réservent. Le 5 juillet 1502, Vincent Bojon, laboureur, signait le bail de quatre mouées de terre gagnables, « la terre des Ruynes ", 43 hectares sur lesquels il s'engageait à faire construire une maison, une grange et une bergerie. Les habitants de la baronnie avaient complètement perdu à la fois leur procès collectif et, en apparence du moins, une bonne part de leur combativitét. Les chanoines avaient su diviser pour mieux choisir parmi leurs dépendants les familles dont les initiatives servaient leur intérêt. 


\section{longue procédure contre les habitants de Parassy ${ }^{76}$ qui prétendaient à l'usage des bois} dits de la Rogerie ; les habitants lui rétorquèrent, en lui rappelant d'abord ce qu'étaient bois pourri, bois mort et mort bois, et ensuite qu'ils avaient le droit de prendre dans ce bel héritage leur usage «en mort bois ne portant pas de fruict: charmes, aulnes, tramble » et qu'ils étaient autorisés aussi d'y mener leur bétail depuis des temps immémoriaux ${ }^{77}$. Là encore trois procureurs furent choisis par les paroissiens et ces derniers eurent gain de cause: Pierre de Contremoret fut condamné à laisser les Parassiens jouir des bois, moyennant un pain et un denier parisis le lendemain de Noël (acte du 10 janvier 1465 n. st.). En 1475 et 1480, les abus paysans, disait Jean de Contremoret le nouveau seigneur, la fermeture unilatérale des bois, lui opposaient les autres, amenèrent à un nouvel accord entre Jean et les habitants. Trente d'abord, puis 25 familles conservèrent leurs usages contre une augmentation des droits, passant désormais à 4 deniers parisis ${ }^{78}$. Si l'on se rappelle qu'un pain est le plus généralement de deux sous, l'augmentation est d'un quart. La victoire, peu onéreuse en fin de compte, de la communauté est davantage liée ici à la faiblesse territoriale et justicière des Contremoret, seigneurs absents pour lesquels 25 pains sont inutiles, qu'à la cohésion des paroissiens.

querelles autour des usages des bois éclataient alors un peu partout. Un grand débat eut lieu entre les dames de Saint-Laurent de Bourges et leurs dépendants de Verneuil ${ }^{79}$, aux portes du Bourbonnais. En mars 1495, trente et un habitants du village durent affronter le prévôt des religieuses et subir une dénonciation en règle de leurs méfaits qui consistaient à « copper, abattre, mener et charroyer, faire copper, abattre et charroyer, vendre en dehors de la dite terre... » les produits des bois abbatiaux. De leur côté les habitants dénonçaient la facilité avec laquelle les dames installaient, en lisière des bois où ils avaient leurs usages, des laboureurs bien décidés, en vertu des formules des baux, à défricher la «terre et la nettoyer des boys et des buissons qui $\mathrm{y}$ sont $"{ }^{80}$. L'attaque juridique, victorieuse, des dames a, de quelques semaines, précédé la mise en lots des parcelles: les religieuses presque ruinées par le grand incendie de Bourges de 1487 qui avait réduit leur monastère en cendres, avaient un besoin si criant d'argent qu'en mai 1495 elles passèrent une trentaine de baux, dont une dizaine comportait en outre une clause d'édification de maison dans une terre à défricher, presque tous pour lots jointifs des usages discutés auparavant. Comme à Graçay, on retrouve dans les preneurs, non seulement des nouveaux arrivants mais quelques-uns des membres de la fabrique que nous connaissons par la reconstruction de l'église paroissiale.

Un peu partout sur la périphérie boisée du Berry, bien davantage que dans champagnes dominées par les immenses domaines affermés ${ }^{81}$, les anciens intérêts collectifs cédaient non seulement devant les appétits individuels, mais aussi en face d'alliances liant seigneurs et paysans, destinées à faire céder les résistances plus ou moins égalitaires des communautés centrales des terroirs en leur opposant les droits particuliers des écarts nouvellement bâtis. Le repeuplement parallèle des cœurs et des marges paroissiales a brutalement confronté les paysans à des cohésions opposées : l'assemblée d'église et les procureurs fabriciens furent parfois en discordance avec les grosses métairies et leurs satellites ouvriers. Les nouveaux venus, récents ou de deuxième génération, devenaient d'année en année les plus nombreux en face des anciennes 
familles survivantes des crises locales. Naguère déracinés, parfois prêts à repartir, les bourgeois de la dernière heure n'éprouvaient plus le besoin de se rassurer au sein des villages, ni au plus proche des maisons fortes ou des petites enceintes des bourgs. Parmi les plus anciennes familles certaines pensaient à leur tour profiter de l'exemple et des occasions pour caser ses propres enfants - et pourquoi pas en les mariant aux nouveaux venus - sur les lisières encore boisées, se détachant ainsi en partie de la cohésion villageoise, voire en jouant sur les deux tableaux. Le bocage devenait au tournant du $\mathrm{XVI}^{\mathrm{e}}$ siècle, la forme neuve de paysage susceptible de convenir aux Berrichons des marges, orphelins volontaires des usages communs, indifférents en apparence aux regroupements paroissiaux.

\section{ANNEXES}

\section{Émeutes contre l'installation d'exploitations sur les communaux}

Interrogatoire de Pierre Garnier, complice des villageois qui ont abattu les haies d'exploitations nouvellement installées de la Chapelle-Montmartin ${ }^{82}$. (Original, Arch. dép. Cher, 8 G 2097, 18 septembre 1492).

A tous ceulx qui ces presentes lectres verront, Jehan Salat, licencié en loix, lieutenant de noble home et saige maistre Pierre de Ganay, aussi licencié en loix, seigneur d'Azy, conseiller du roy nostre seigneur et son bailly de Berry, salut. Savoir faisons que aujourdhuy nous nous sommes transportez es prisons de la chambre des comptes du roy nostre seigneur à Bourges, par devers et a la personne de Pierre Garnier, prisonnier esdites prisons, aagé de XL ans ou environ, de la paroisse de la Chappelle Moynemartin, a nous renvoyé par messeigneurs de la court de Parlement pour proceder a la pugnicion des exces, monopoles et assemblees faictes contre les officiers du roy par lui et autres ses aliés et complices, ainsi que verrons estre affaire par raison, nonobstant opposicion ou appellacion quelzconques jusques a sentence diffinitive, inclusive de mota execucione s'il en est appellé, et pour iceulx faire et parfaire le proces dudit prisonnier, lequel prisonnier, apres le serment par luy faict de dire verite, nous a dit et confessé sans aucune contrainte, en presence d'honorable homme et sage maistre Giles Pain, procureur general du roy nostredit seigneur ou dit bailliage, et de Guillaume Chaumeau, clerc notaire de la cour de Bourges et commis du greffier dudit bailliage, que ou mois de janvier dernier passé, autrement du jour ne s'est recordé, Jehan Garnier son frere l'envoya querir par un petit garçon son filz, lequel luy dist qu'il allast devant l'eglise de la dite Chapelle Moynemartin ou estoit ledit Pierre Garnier son frere et plusieurs des habitans dudit lieu, lequel prisonnier y alla et quant il fut devant ladite eglise, il trouva ledit Jehane Garnier son dit frere, ung nommé Estienne Salvet, Jehan Bezard, Jehan Gallette, Jehan de Bourges, Guillot et Colin Bedaulx, freres, Jehan Bedaulx, filz de Jehan Bedaulx, Jehan Thierry et Jehan Thierry, fils de Estienne Thierry, Denis Bezard, Jehan Mesnil et aultres des noms desquelz il ne s'est recordé, tous lesquels et ledit prisonnier, apres que ledit Salvet leur eut dit qu'il estoit venu de Paris et qu'il avoit trouvé par conseil que ilz devoient garder certain arrest de la court de 
Parlement qui avoit esté donné a leur prouffit contre les venerables de la Saincte Chapelle du pallaiz de Bourges, conclurent qu'ilz yroient rompre et abatre les hayes qu'avoient faictes Guillaume Moreau, Guillaume Dupuy, Jehan Pichon, Estienne Gallier, et autres ; et, de fait, incontinent apres la dite conclusion prise, luy, touz les dessusditz et autres jusques au nombre de vingt habitans dudit lieu de la Chappelle Moynemartin allerent rompre et abatre lesdites hayes des dessusdits Dupuy, Gallier, Pichon et Moreaul, lesquelles hayes ilz avoient faictes es heritaiges qu'ils tiennent desdits venerables du Pallais, combien qu'ils soient usagiers communaulx ausditz habitans, ainsi que disoient ledit prisonniers et sesdits compaignons ; et estoient enbastonnez, c'est assavoir ledit prisonnier d'une forche de boys, ledit Jehan Thierry d'un espieu, ledit Guillaume Bedaulx d'une congnye et ung autre, du nom duquel il ne s'est recordé, d'un espieu et plusieurs autres de congnyes, pour rompre lesdites hayes; et apres qu'ils les eurent rompues, s'en allerent chascun en leur maison. Et depuis fut constitué prisonnier par Anthoine Amy, sergent royal, par vertu de la commission de monseigneur le bailly, ou de nous, et troys autres de sesdits compaignons, c'est assavoir lesditz Denis Bezard, Jehan Thierry et Jehan Viguier, et les amenoit prisonniers le dit Amy en ceste ville de Bourges. Et quant ils furent a Preuilly" en l'ostel d'ung nommé Cavet Euvrardet audit lieu, Jehan Bezard, Pion Chambrias, Jehan Mesnil dit Courtin, Guillaume Bedault dit Darme, Colin Bedault dit Darme, Jehan de Bourges, Guillaume Viguier, Jehan Robin, Loys Salvet, Loys Mace, Jehan Thierry, fils de Estienne Thierry, Pasquet Mesnier et plusieurs autres ne scet jusques a quel nombre, parce qu'il ne les vit pas trestous, lesquels dirent au dit sergent qu'ilz vouloient avoir lesditz prisonniers qu'il emmenoit ; et en parlant ensemble, maistre Estienne Dupont sortit hors de la chambre ou ils estoient et apres lui s'en sortirent lesditz Jehan Thierry et Denis Bezard, prisonniers, et ledit Viguier s'en sortit par la fenestre, devant laquelle y avoient le dit Bezard et austres qui demandoient tousjours lesditz prisonniers audit Amy, lequel leur respondit quilz estoient prisonniers du roi, et que s'ils estoient saiges ilz ne les lui ostereoient pas et qu'ilz se mectoient en dangier. Interrogé si ledit Jehan Bezard ou autre de ses compaignons osterent pas par force lesditz prisonniers audit Amy, et si ledit Bezard lui bailla pas un coup de forche sur le bras et un coup de javeline tellement qu'ilz le blesserent en la main jusques a grant effusion de sang pource qu'il ne vouloit lascher le dit Viguier, lequel ilz vouloient tirer a force par la fenestre, dit que non ; bien vit le dit Amy qui seignoit de la main ung peu durant ledit debat, mays ne scet qui luy avoit fait. Interrogé si tous les dessus ditz qui furent à la recouvre desditz prisonniers, ou aucuns d'eulx, disoient pas au dit sergent qu'ilz auroient et leur rendroit lesditz prisonniers de bon gré ou par force, en jurant, blasfemant et regnyant Dieu, dit que non ${ }^{+}$. Interrogé pourquoi les dessusditz habitans de ladite Chappelle Moynemartin ne recouvrerent ne emmenerent ledit prisonnier comme les autres, dit que ce fut pource qu'il ne s'en voult pas aller et sortir de ladite chambre car il cognoissait bien qu'il et sesditz compaignons avoient tort d'eulx en aller, et aussi ceulx qui les recouvroient et emmenoient, et ne monta, ne fut monté par le dit Amy, ne autre, dans la chambre haulte de ladite maison dudit Cavet, affin qu'il ne fust recours et emmené par les dessusditz, et ne bougea de la chambre basse ou il et ledit Amy et sesditz compaignons prisonniers avoient toujours esté. Interrogé par quelle auctorité et puissance ilz firent la dite assemblee pour aller rompre lesdites hayes, et qui leur avoit donné congie d'eulx assembler, dit que ilz firent ladite assemblee de leur auctorite privee, sans congie et auctorite de justice, et auparavant n'en avoient point fait. Interrogé si, en rompant lesdites hayes, lui et ses dits compaignons et complices qui étaient a rompre ne 
foullerent et ne gasterent pas les bles de ceulx a qui estoient lesdites hayes, en jurant et blasfement et regniant Dieu et les voulant battre pource qu'ils leur contredisoient, et vouloient empescher qu'ilz ne les rompissent, et leur disoient pas qu'ilz et leurs biens estoient en la saulvegarde du roy, laquelle leur avoit este notifiee, en se moquant et disant par desrision : " Trupt, trupt, on ne vous touche, tu nes pas roy ne royne ", dit que non, et dit que si un de ceulx a qui estoient lesdites hayes eust dit audit prisonnier qu'ilz estoient en la saulvegarde du roy, il n'eust point aydé a abatre lesdites hayes. Interrogé si dist pas a Jehan Lore dit Pichon telles ou semblables parolles « va villain tes seigneurs ne sont que moynes et maugre eulx nous ferons hayes a chasser les serfs et biches ", dit que non ; et furent lesdites hayes rompues depuis l'arrest donné pour ces habitans contre les venerables et executé par un de nos seigneurs de ladite court, duquel il ne scet le nom. Interrogé si, depuis l'execution dudit arrest, il et sesdits complices dudit lieu de la Chappelle Moynemartin ont pas esté on boys de Ruynes, appartenant ousditz venerables, et d'ycellui transporté certaine quantite de boys que lesditz venerables avoient venduz à des charbonniers, dit qu'il n'y a point esté, et ne scet point que aucun desditz habitans y aye esté. Interrogé si des choses dessusdites et chascune d'icelles il s'en veult rapporter a Perette, vesve de feu Estienne Daguenet, Martin Besson, Pasquette, vesve de feu Jehan Petion, Jehan Lore dit Pichon, Guillaume Dupin, Thomas Lore dit Pichon, Estiennete, femme de Guillaume Gallier, Plaisance, femme de de Jehan Moireau, frere Jehan de la Vallee, Guillemette, vesve de feu Martin Menart, Michau Moireau, Jehan Habert, Jehan Besson, et frere Guillaume Pecton, dit que non parce qu'ilz sont adverse partie et qu'ilz vouldroient qu'il et autres desdits compaignons et complices feusssent destruictz, et au regard dudit Martin Besson, il ne le congnoist. Fait a Bourges, par nous lieutenant commis dessus dit, et donné sous le seel es causes dudit bailliage en tesmoing de ce, le XVIIII ${ }^{e}$ jour de septembre. L'an mil CCCC quatre vingt et douze.

[signé] Chaumeau, pour le greffier.

+ qu'il oyst pource qu'il est sourt et entend dur, comme cy dessus

Évolution décennale des tailles et des bourgeoisies de la seigneurie de Graçay (1436-1493)

(Les pourcentages se lisent horizontalement)

\begin{tabular}{|l|l|l|l|l|l|}
\hline Années & Bourgeoisies & $\%$ & Taillables & $\%$ & Total \\
\hline \hline 1436 & 144 & 61,8 & 89 & 38,2 & 233 \\
\hline \hline 1446 & 182 & 62,8 & 198 & 37,3 & 380 \\
\hline \hline 1456 & 221 & 68 & 104 & 32 & 325 \\
\hline \hline 1461 & 376 & 76,9 & 113 & 23,1 & 489 \\
\hline \hline 1466 & 552 & 81,7 & 124 & 18,8 & 676 \\
\hline \hline 1470 & 655 & 85,6 & 110 & 14,4 & 765 \\
\hline
\end{tabular}




\begin{tabular}{|l|l|l|l|l|l|}
\hline 1481 & 756 & 90,7 & 78 & 9,3 & 834 \\
\hline \hline 1493 & 781 & 92,2 & 67 & 7,9 & 848 \\
\hline
\end{tabular}

\section{NOTES}

1.E. Chénon, « L'ancien coutumier du pays de Berry (XIVe-XVe siècles) », extrait de la Nouvelle Revue du Droit Français et Étranger, 1916, 32 p. L'auteur a décelé dans les articles du Coutumier du XVI ${ }^{e}$ siècle, un premier état rédigé vers 1313, un second, œuvre de Jean de la Loë, vers 1433, et enfin la rédaction de 1536.

2.Il n'est pas question ici de refaire la très remarquable introduction de J. Hilaire, « Le village, la coutume et les hommes ", La coutume au village dans l'Europe médiévale et moderne, Flaran XX, 2001, pp. 7-17.

3.On ne saurait accepter les yeux fermés les anachronismes de l'essai de G. Bois, $L a$ grande dépression médiévale XIV et $X V^{e}$ siècles. Le précédent d'une crise systémique, Paris, PUF, 2001, pourtant riche de quelques rappel salutaires en matière économique, que l'on peut aussi trouver ailleurs (P. Contamine et alii, L'économie médiévale, Paris, Armand Colin, 1993, p. 329-395).

4.G. Thaumas de la Thaumassière, Coutumes locales de Berry et celles de Lorris commentées, Bourges, Toubeau, 1689, p. 195 (1268). L'auteur ensuite sera abrégé : La Thaumassière, Cout. loc. Lignières c., ar. Saint-Amand-Montrond, Cher.

5.La Thaumassière, Cout. loc., p. 86 (1278) : manumittimus ab omni jugo servitutis et ab omni servitio, tallia, consuetudine seu quacumque exactione indubita.

6.Arch. dép. Cher, $8 \mathrm{G} 2101$ (1466-1467), fol. 32v : Nos autem manumissiones et libertates predictas[...] volumus et concedimus de voluntate nostre. La copie faite ici de la charte de Pierre de Graçay est celle qui fut confirmée en juillet 1246 par saint Louis - scellée « en queue double de soye en cire verd »- . Elle présente l'intérêt de montrer que cette confirmation fut onéreuse : la baronnie devrait désormais à chaque Saint-Michel porter quatre muids d'avoine au grenier royal d'Issoudun.

7.Le cas me paraît clair pour la charte de Montry, mansus du prieuré de Sainte-Cécile (ancienne paroisse, act. com. et c. Saint-Christophe en Bazelles, ar. Issoudun, Indre) alors dépendant de Déols : vers 1150 Renaud de Graçay a abandonné les coutumes quas male tenebat, [...] ita quod quicumque in mansum supradictum habitare voluerit est liber ab omni expedititione et ab omni servitio. Le doute que l'on pourrait avoir sur le sens classique de servitium : servitude, ou médiéval : service militaire est levé par le contexte car Renaud a restitué le manse à Déols in liberum allodium. Il s'agit bien ici de dégager le prieuré des charges militaires envers un seigneur châtelain et non de la liberté personnelle et individuelle des habitants du manse, ces charges militaires ayant pu peser sur les libres comme sur les serfs (Arch. dép. Cher, 2 F 52 n 4).

8.La Thaumassière, Cout. loc., p. 91 : pour Bengy-sur-Craon (1257), par exemple, en 1333 et $1439 \mathrm{cf}$. infra.

9.Ibid., p. 90, à Saint-Chartier (1251), et p. 184, à Châteaumeillant (1220) où, hors des limites de la franchise, ceux qui relèvent du dominium, devront verser pour garder leur héritage une somme de 5 sous au seigneur, mais s'ils en sont incapables un arbitrage pourra amenuiser la somme. 
10.Il est inutile de répéter ici ce qu'a énuméré G. Devailly, Le Berry du $X^{e} a u X V^{e}$ siècle, Paris-La Haye, 1973, p. 538 à 544.

11.R. Fossier, « Franchises rurales, franchises urbaines... », Villes, bonnes villes, cités et capitales, Mélanges offerts à Bernard Chevalier, Tours, 1989, p. 187.

12.Mention particulière du castrum aux Aix-d'Angillon, Châteaumeillant, Graçay,

13.Par exemple le bourg de Preuilly (c. Lury-sur-Arnon, Cher).

14.Croix de franchises à Châteaumeillant, Graçay, Preuilly, Boisroux, Dun, Gournay, Barlieu, Le Pin.

15.Le terme usuel est metas : au Châtelet, à Saint-Chartier

16.Le « circuit » est précisé aux Aix, à Châteauneuf-sur-Cher, Orsennes, Saint-Benoîtdu-Sault, Culan et dans un dénombrement de Gargilesse.

17.Au Châtelet, Guillaume III de Chauvigny accorda en 1299 des franchises aux hommes manentes et mansuri amodo in ville de Castelleto et infra metas et plus loin il concèda aux habitants que Gaufridus à la Charnée et ejus heredes de dicta libertate sint, dum tamen extra dictas metas faciant mansionem. (La Thaumassière, Cout. loc., p. 142, avec une mauvaire transcription (infra pour extra, qui rend la faveur faite à Geoffroi a la Charnée incompréhensible). Le Châtelet, c., arr. Saint-Amand-Montrond, Cher.

18.Ibid., p. 538, 539 (deux fois).

19.Pour la seule seigneurie de Graçay dont il sera question plus loin, entre 1420 et 1465 on a pu repérer 272 partages entre les chanoines de la Sainte-Chapelle de Bourges et 35 seigneurs environnants. Ils ont touché le nombre non négligeable de 829 personnes. Ces partages concernent aussi bien de vrais serfs que des gens réputés francs : ces "bourgeois », qui jouissant de privilèges de liberté subissent aussi des conséquences personnelles inégalitaires au sein de leur famille en raison des caractères héréditaires de l'achat de la liberté .

20.La Thaumassière, Cout. loc., p. 156 (Châteauneuf, 1258) : si forte non extarent heredes, dicti quatuor jurati dicta bona saisirent, tenerent et custodirent usque ad annum et diem; et si tunc non apparueret heres ipsi quatuor mobilia et quintam partem immobilium in pios usus converterent [...] ; residua vero immobilia, domino Castri novi remanebunt, donec appareat heres cui de jure successio debeatur.

21.ORF, XI, p. 363 : Excubice autem, more quo prius, fient, admonitu tamen boni viri...

22.La Thaumassière, Cout. loc., p. 140 : Decem viri quos Canonici singulis annis de majoribus villae et melioribus eligent...

23.Idem, pp 91-94, et Arch. dép. Cher, 8 G 1239.

24.F. Bouthault, Les domaines du chapitre de Bourges au début du XIII ${ }^{e}$ siècle, Thèse de droit, Paris, 1942, pp. 24-25 et 57. En 1301, les revenus en deniers de la prévôté de Bengy représentaient deux fois ceux de la fortune proprement berruyère du chapitre (Arch. dép. Cher, 8 G 825, compte de 1301-1302 pour les prévôtés de Bourges et Bengy).

25.BnF, n. acq. lat., 1274 , fol $344 \mathrm{v}-345$.

26.En 1565 Bengy n'avait plus que 112 feux. (cf., J. Dupâquier, Statistiques démogaphiques du Bassin parisien, Paris, Gauthier-Villars, 1977, p. 111, d'après BNF, m. f., 3198, Etat des feux du Berry),

27.La Thaumassière, Cout. loc., p. 91 : dictce parrochice homines unanimi assensu et voluntate procuraores suos constituerunt.

28.Germigny l'Exempt (c. La Guerche-sur-l'Aubois, ar. Saint-Amand-Montrons, Cher) relevait au Moyen Âge du Bourbonnais et du doyenné de Montfaucon, lui aussi entièrement en Bourbonnais, la cure était à la nomination de l'archevêque de Bourges, ici arbitre de la transaction. 
29.La Thaumassière, Cout. loc., p. 92 : la taille était rachetée 30 livres, les deux charrois annuels 6 livres, les avenages 100 sous tournois, le fouage 20 sous, la mortaille 30 livres, les trois corvées 60 sous.

30.Idem., p. 92 : Eligentur autem quolibet anno ab hominibus parrochice de Bangiaco, vel a majori parte ipsorum, infra festum Johannis Baptistee, septem boni viri, fideles et non suspecti. 31.Idem, p. 93. Il va de soi que ces représentants devaient être boni homines fideles et non suspecti. Ils ne devaient pas molester les justiciables. En outre trois d'entre eux devaient porter les redevances aux chanoines dans leur palais de Bourges, distante de $25 \mathrm{~km}$. 32.La Thaumassière, Cout. loc., p. 94.

33.Arch. dép. Cher, 8 G 825 (rouleau pour 1301-1302), VI . Les tailles en question représentent $14 \%$ de la recette de Bengy.

34.Arch. dép. Cher, 8 G 1239. Procuration du mercredi avant l'épiphanie 1333 (=5 janvier 1334) et transaction du mardi après la Saint-Guillaume d'hiver 1333 (= 11 janvier 1334 ; la Saint-Guillaume, fête du saint évêque de Bourges, était fêtée dans l'ancien bréviaire du diocèse de Bourges le 10 janvier).

35.Arch. dép. Cher, 8 G 1239 (17 septembre 1439).

36.Arch. dép. Cher, 8 G 752 (1444-1445), fol. 9v-10v : relevé des froments et fol. 36-37 relevé des tailles en deniers. On dénombre 2 tailles à 4 boisseaux, 6 à 2 boisseaux et 23 à 1 boisseaux ( $74 \%$ de l'ensemble).

37.En 1450, il y eut 49 taillables, en $1465: 71$; en $1475:$ 99, enfin, 117 en 1482.

38.La région de Graçay, en "Boischaut nord ", se situe entre la bordure nord-ouest de la Champagne de Vatan et la vallée du Cher, dans une zone vallonnée et boisée. 39.Nous sommes très loin des chiffres repris par A Leguai d'une estimation des serfs bourbonnais des châtellenies de Montluçon, et Murat pour la « fin du XVe s » qui propose plus de $20 \%$ de serfs, en revanche nous nous rapprochons dans les années 1470 de ce qui est suggéré pour Chantelle : 11,95 \% (cf. A. Leguai, « Le servage en Bourbonnais aux XIV et $\mathrm{XV}^{\mathrm{e}}$ siècles ", Cahiers d'Histoire, t. XX, 1975, nº 1, p. 29). 40.Genouilly, c. Graçay, ar. Vierzon, Cher.

41.Arch. dép. Cher, 8 G 2222 : le 12 janvier $1489(=1490)$ Jean Mesny prend à bail perpétuel 18 septérées (soient 17 ha environ) des terres enn bois et buissons « dans lesquels les hommes de la serve condition de messeigneurs avoient usaige, lesquel ont este manumis et affranchis moyennant qu'ils ont renonce audit usaige ».

42.La Chapelle-Montmartin, c. Mennetou-sur-Cher, ar. Romorantin-Lanthenay, Loir-etCher.

43.Arch. dép. Cher, 8 G 2102 (1466-67), fol. 147 : « De Jaunet Vachier, ung arpent de pré au pré de la Planche audessus de la Chapelle [Montmartin] en laquelle il a assis $10 \mathrm{~s}$.t. de rente perpetuelle a cause de sa manumission ». La constitution de rente, conservée, est du 15 mai 1466, Jaunet y est dit « honnête homme », reconnaissance d'aisance sociale qui explique peut-être sa manumission.

44.La crise éclata quand les chanoines lotirent les bois en exploitations défensables: Arch. dép. Cher, 8 G 2097 (1491-1492) cf. infra.

45.Anjoin, c. Saint-Christophe-en Bazelle, ar. Issoudun, Indre.

46.Arch. dép. Cher, 8 G 2162 (1479-80), fol. 65-67v , le compte énumère les bourgeois de la franchise regroupés dans les onze hameaux et domaines, et fol. 70-71v , les 79 lopins de vigne, propres « aux gens de Vonne ", regroupés en vignobles (cf. F. MichaudFréjaville, «Vignerons des villes et vignerons des champs en Berry et Orléanais à la fin du Moyen Âge ", Vignerons, Bulletin du Centre Pierre Léon d'histoire économique et sociale, 1996, n 3-4, pp. 68-69). 
47.Arch. dép. Cher, $8 \mathrm{G} 2145$, fol. $140^{\mathrm{v}}$ : le receveur remet en dépense 90 l. t. sur les 120 1. t. qu'il avait portées en recette " parcequ'[il] n'a peu recepvoir autre chose des dictes tailles et franchises obstant le proces qui est entre messeigneurs et les habitans de la baronnie ".

48.Arch. dép. Cher, 8 G 2119, fol. $12^{\mathrm{v}}-16^{\mathrm{v}}$. et fol. 31-31ㄴ. Taillables : Vincent Rivière (il paie un denier pour son chesal de Neilly, paroisse de Genouilly, et 5 deniers obole pour les cinq terres, vignes et bois énumérés à la suite) et André Gillet, au même hameau. Sont bourgeois de Gracay quatre membres de la famille Le Seurre, Etienne Jagaut (le boucher du lieu) et Robert du Moustier.

49.Idem., fol. 20. Ces cens étaient partagés entre le duc de Berry (plus tard les chanoines de la Sainte-Chapelle) et le prieur de la Chapelle-Montmartin (dépendant de SaintMartin de Tours et qu'on appellait alors la Chapelle Moynemartin). Une partie des censitaires devait relever du prieuré, cependant, et curieusement, nous n'avons aucune trace au $\mathrm{XV}^{\mathrm{e}}$ siècle de mariage ayant nécessité une redistribution des dépendants entre les deux seigneurs. Il n'y a d'ailleurs que sept « partages » de dépendants concernant La Chapelle, sur les 272 opérés entre 1423 et 1466 entre les chanoines de la Sainte-Chapelle et 36 autres seigneurs, enclavés ou jouissant d'un droit de suite dans l'ensemble de la terre de Graçay (d'après Arch. dép. Cher, 8 G 2103).

50.F. Michaud-Fréjaville, « Bourgeois ruraux, serfs barons, un petit peuple du duché de Berry à la fin du Moyen Âge ", Le petit peuple dans l'occident médival, terminologie, perceptions, réalités, Actes du congrès international tenu à l'Université de Montréal, 18-23 octobre 1999, Paris, Publications de la Sorbonne, 2002, pp 251-263.

51.Arch. dép. Cher, $8 \mathrm{G} 2103$ (1466) : ces presque six cent personnes arrivées de fraîche date ne représentent cependant que $6,5 \%$ de la population connue pour avoir vécu dans la seigneurie pendant cette période.

52.Et parmi les récentes A.Girardot, Le droit et la terre, le Verdunois à la fin du Moyen Âge, Nancy, Presses Universitaires, 1999, t. II, pp. 778- 804 : « La terre aux ruraux ».

53.E. Carpentier, M. Le Mené, La France du XI ${ }^{e}$ au XVe siècle, Paris, 1996, p. 477 : « Face aux communautés villageoises, ils tentèrent de renforcer leurs droits en restreignant l'étendue des pâtis, en pratiquent des enclosures et en limitant l'usage des bois ». 54.Saint-Loup, c. Mennetou-sur-Cher, ar. Romorantin-Lanthenay, Loir-et-Cher. Les chanoines avaient à Saint-Loup une quinzaine de bourgeois payant leur franchise au milieu du XV siècle. Le château de Sauveterre se situe à $2 \mathrm{~km}$ à l'ouest du village au sommet du revers de la rive gauche du Cher, 55.Dun-le-Poëlier, c. Saint-Christophe-en-Bazelle, ar. Issoudun, Indre.

56.Arch. dép. Cher, 8 G 2221 (6 mai 1448) : le rendez-vous était à Sainte-Catherine-duFeust, prieuré de Déols, dans les actuelles " bruyères de Sainte-Catherine », commune de La Chapelle-Montmartin (c. de Mennetou-sur-Cher, arr. de Romorantin-Lanthenay, Loir-et-Cher).

57.Arch. dép. Cher, 8 G 2119, fol. 24 (1372-1373), c'est le texte auquel se référèrent les deux parties pour le procès qui eut lieu en 1491. C'est une des raisons de la conservation jusqu'à nos jours du document $:$ il donne l'état de la seigneurie l'année où Jean de Berry en prit possession. La zone décrite est celle entre Fouzon (ici le Foron) et Cher.

58.F. Michaud-Fréjaville, «Baux et servage en Berry », Colloque de la Société Histoire et Sociétés rurales, Angers, septembre 1997 (sous presse).

59.La Thaumassière, Cout. loc, p. 328, Coutume de Bourges, Titre X : Coustumes prædiales, VI : «Par la coustume, es lieux incultivés qui sont en chaulmes, en friches, 
en bruyeres et buyssons, n'y a et n'y chet prinse de bestes pour les mener a justice en quelque temps que ce soit $»$.

60.Arch. dép. Cher, $8 \mathrm{G} 2222$. Bail à charge de bâtir une maison de quatre toises $(7,74 \mathrm{~m}$ de long, ce qui est relativement modeste,) avant quatre ans, le cens figure en 1480.

61.Arch. dép. Cher, $8 \mathrm{G} 2164$, fol. $106^{\mathrm{v}}(1486)$ : quatre baux nouveaux de bois et buissons sont ajoutés pour cette seule année à la rubrique de La Chapelle-Montmartin :

Guillaume du Pain, Colin et Guillaume de Rancé, Etienne Gallier, Jean, Pierre et Louis Salvet.

62.Arch. dép. Cher, $8 \mathrm{G} 2222$. Bail de 8 sextérées de terres jouxtant les bois de l'abbaye cistercienne d'olivet, ceux de la Touche et ceux des chanoines, moyennant la construction d'une maison à une ferme et une croupe dans les trois ans.

63.Arch. dép. Cher, 8 G 2103, fol. 244 : le partage entre les Chippault dépendants de Déols et ceux des la Sainte-Chapelle avait eu lieu en 1448.

64.Arch ; nat., JJ 222, fol. 110, n 244.

65.Arch. dép. Cher, $8 \mathrm{G} 2097$, arguments pour la plaidoirie des chanoines, fol. $6^{\mathrm{v}}$.

66.Arch. dép. Cher, 8 G 2165 (1490-1491), fol. 139-140v : refus de payer les tailles et les franchises de Graçay.

67.Arch. dép. Cher, $8 \mathrm{G}$ 2097, original latin. Idem, copie et traduction par les officiers des chanoines. Les pièces du dossier ne sont pas numérotées.

68.Arch. dép. Cher, 8 G 2097 : registre non numéroté de 49 folios.

69.Giroux, c. Vatan, ar. Issoudun, Indre, en limite de Champagne berrichonne, le domaine était une acquisition des chanoines.

70.Arch. dép. Cher, 8 G 8 G 2101 (1465), fol. 137 et 141. La métairie avait été prise par Jean Peton.

71.Idem, fol. 147 : depuis 1458 Macé Salvet avait pris une partie de la Tranchée à charge de bâtir une maison et des terres « à la Marchouère » aujourd'hui encore mitoyen du terroir des « Grands pâtureaux ».

72.Pièce justificative.

73.Arch. dép. Cher, $8 \mathrm{G}$ 2097, fol. $6^{\mathrm{v}}$ : « [...] Ils ont fait l'absolution de XXXVI personnes qui avoient confessé avoir coupé du bois ». Les remises d'amende sont en effet aussi nombreuses que les voyages pour procès dans la comptabilité des chanoines.

74.Arch. dép. Cher, $8 \mathrm{G}$ 2222. Bail perpétuel moyennant 16 septiers de blé, seigle avoine, marsèche et 6 sous tournois portant vente.

75.Arch. dép. Cher, 8 G 2164.

76.Parassy, c. Les Aix-d'Angillon, ar. Bourges, Cher.

77.Arch. dép. Cher, E 876, (10 janvier 1465 n. t.). Idem, 1480 : copie informe du XVI ${ }^{\mathrm{e}}$ siècle.

78.Idem, copie collationnée du 10 octobre 1654.

79.Verneuil, c. Dun-sur-Auron, arr. Saint-Amand-Montrond, Cher,

80.Arch. dép. Cher, 39 H 218. Des baux ont été passés en particulier avec des habitants de Thaumiers (c. Charenton-du-Cher, ar. Saint-Amand-Montrond, Cher).

81.F. Gay, La champagne du Berry, Bourges, Tardy, 1969, Cette thèse de géographie montre parfaitement, dans une longue partie historique, l'affermissement de la grande propriété où céréales et élevage ovin s'associent de plus en plus étroitement à partir du $\mathrm{XVI}^{\mathrm{e}}$ siècle sur de vastes terroirs ouverts.

82.La Chapelle Montmartin, c. Mennetou-sur-Cher, ar.Romorantin-Lanthenay, Loir-etCher.

83.Preuilly, c. Lury-sur-Arnon, ar. Bourges, Cher. 


\section{AUTEUR}

FRANÇOISE MICHAUD-FRÉJAVILLE

Université d'Orléans, E. A. 2436 « Les territoires de l'identité » 